\title{
Cytotoxic Lesions of the Corpus Callosum Caused by Thermogenic Dietary Supplements
}

\author{
(D).A. Galnares-Olalde, (D)A.J. Vázquez-Mézquita, (D) G. Gómez-Garza, (DD. Reyes-Vázquez, (DV. Higuera-Ortiz, (D)M.A. Alegría-Loyola,
} and (D) A. Mendez-Dominguez

\begin{abstract}
SUMMARY: Consumption of over-the-counter dietary supplements to reduce body weight is common among the population. Thermogenics are herbal combinations that claim to produce a fat-burning process through an increase in the cellular metabolic rate and greater cellular energy consumption, having a high risk for patients developing toxic leukoencephalopathy. We present a series of 6 patients with acute neurologic symptoms and MR imaging showing restricted diffusion and decreased apparent diffusion coefficient values (mean value, $400 \mathrm{~mm}^{2} / \mathrm{s} \times 10^{-6}$ ) in the entire corpus callosum compatible with a cytotoxic lesion of the corpus callosum. Although patients responded favorably to the product discontinuation with rapid recovery of neurologic symptoms, there was a more prolonged resolution on imaging alterations. Because of the widespread availability and unregulated nature of thermogenic dietary supplements, physicians must be aware of the clinical and radiologic characteristics of these potential complications of their use.
\end{abstract}

ABBREVIATION: CLOCC $=$ cytotoxic lesion of the corpus callosum

C onsumption of over-the-counter dietary supplements to reduce body weight is common among the population. ${ }^{1}$ Thermogenics are natural herbal combinations that aim to augment fat-burning by producing an increase in the cellular metabolic rate and greater cellular energy consumption. These supplements are available in most countries and can be purchased over the Internet without a prescription. Although local drug-regulation agencies have warned against the indiscriminate use of these products, they are still widely available under various brand names. ${ }^{2,3}$

Recently, there have been reports of severe-yet-reversible toxic leukoencephalopathy related to the intake of thermogenic supplements. ${ }^{3}$ Characteristic MR imaging findings reveal intramyelinic edema that has a predilection for the corpus callosum. These MR imaging changes have been recently termed "cytotoxic lesions of the corpus callosum" (CLOCCs). ${ }^{4}$ Previously, similar findings were described as mild encephalitis or encephalopathy with reversible splenial lesions and reversible splenial lesion syndrome

Received February 15, 2019; accepted after revision May 23.

From the Internal Medicine Service (J.A.G.-O.), Radiology Service (A.J.V.-M., G.G.-G., D.R.-V.), Department of Rheumatology (V.H.-O.), and Neurology Service (M.A.A.-L., A.M.-D.), The American British Cowdray Medical Center, Mexico City, Mexico. J.S. Galnares-Olalde and A.J. Vázquez-Mézquita contributed equally to this work.

Please address correspondence to Gilberto Gómez-Garza, MD, The American British Cowdray Medical Center, Avenida Carlos Graef Fernández 154, Cuajimalpa, Tlaxala Santa Fe, 05300 Mexico City, Mexico; e-mail: ggomezgarza@gmail.com

http://dx.doi.org/10.3174/ajnr.A6116

among others. However, not all cases are mild, reversible, or limited to the splenium. ${ }^{3,4}$

It is believed that these lesions are a result of an inflammatory cascade of changes manifested as cytotoxic edema secondary to an increased influx of water molecules in astrocytes and neurons caused by the release of the inflammatory cytokines interleukin-1 and interleukin-6. These interactions create feedback loops that increase extracellular glutamate levels. The increased quantity of extracellular glutamate has an excitatory effect at membrane receptors such as the $\alpha$-amino-3-hydroxy-5-methyl-4-isoxazolepropionic acid (AMPA) and N-methyl-D-aspartate (NMDA) receptors, as well as aquaporins and $\mathrm{Na}^{+} / \mathrm{K}^{+}$pumps. The most affected region of the corpus callosum is the splenium due to its higher density of glutamate and cytokine receptors. ${ }^{4}$ The purpose of this research was to contribute to the understanding of toxic causes of CLOCCs, to describe the clinical and MR imaging findings of CLOCCs caused by thermogenic dietary supplements, and thus to expand the body of literature on the diagnosis of this condition.

\section{Case Series}

This case series report was performed under institutional review board approval. An observational, descriptive, retrospective study was conducted of both inpatient and outpatient subjects during a 3-year period. We searched the registry of the hospital for cases with clinical and brain MR imaging findings compatible with toxic leukoencephalopathy that reported previous use of thermogenic dietary supplements. As inclusion criteria, we considered 


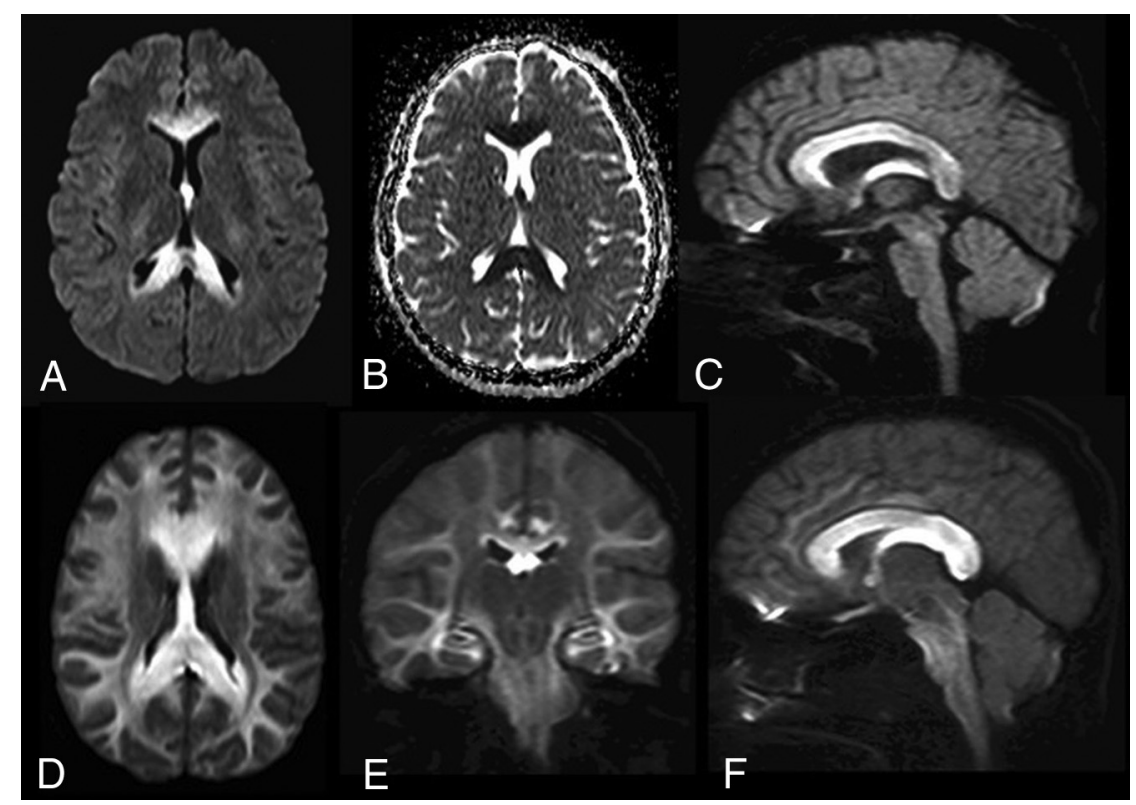

FIG 1. CLOCCs caused by thermogenic dietary supplements. Axial DWI $(A)$ and corresponding $A D C$ map $(B)$ of case 1 show restricted diffusion representing cytotoxic edema at the corpus callosum. C, Sagittal DWI shows a hyperintense corpus callosum, cerebral commissures, and fornix in case 2. D and $E$, Axial DWI and corresponding ADC map from case 4 show more extensive cytotoxic edema that affects the entire corpus callosum and cerebral white matter. $F$, Sagittal DWI of case 5 reveals an enlarged and edematous corpus callosum, fornix, and cerebral commissures with involvement of the peripheral cerebral white matter, cerebral peduncles, and brain stem, along with the optic nerves.

those 18 years of age and older who had a history of thermogenic supplement intake during the previous 6 months, acute neurologic symptoms at presentation, and white matter brain alterations on MR imaging, characterized as diffusion-weighted imaging $(b=1000)$ restriction with decreased apparent diffusion coefficient values. Six patients fulfilled the criteria and were included in this series; all of them were women. The mean age was 33 years (range, 23-43 years); all of the patients had normal body mass index values (mean, 21.01; range, 19.2-23.7) at the time of the examination. None were obese or significantly overweight before starting use of the dietary supplement.

Of the patients, one had a history of social alcohol consumption, 2 were active smokers, one was receiving an antiepileptic drug (phenytoin) and an anticoagulant (acenocumarol) for a previous ischemic stroke, and another was on antidepressant medication (amitriptyline). Regarding the dietary supplement intake, the median time of consumption for the 6 patients was 5.3 months (range, 1-18 months); all of them took the recommended dosage of the product.

At the time of the medical evaluation, headache was present in 5 patients; generalized weakness, in 4; distal paresthesia, in 3; blurred vision, in 3; photophobia, in 2; and phonophobia in 1. The clinical spectrum was nonspecific, ranging from normal examination findings to gait disturbances.

On MR imaging, restricted diffusion with low ADC values (mean, $400 \mathrm{~mm}^{2} / \mathrm{s} \times 10^{-6}$; range, $194-550 \mathrm{~mm}^{2} / \mathrm{s} \times 10^{-6}$ ) was present in the corpus callosum and cerebral commissures of all 6 patients (Fig 1), while the hemispheric cerebral white matter, brain stem, and dentate nucleus of the cerebellum were affected in 3 (Fig 2). The optic nerve was affected in 3 patients. The signal alteration was less evident on the FLAIR sequence as a subtle hyperintensity in only 3 patients.

After suspending the dietary supplement intake, all neurologic symptoms resolved within a mean time of 2.8 days (range, 1-5 days). A total of 4 patients underwent control MR imaging studies. While 1 patient had complete resolution in the imaging findings at 6 weeks, 1 patient did not show improvement on imaging at 2 months. Only partial resolution of the MR imaging alterations showed in a 10-month control study for 1 patient, and 1 had complete resolution 22 months after the initial MR imaging. Demographic and MR imaging findings are listed in the Table.

\section{Patient Presentation}

Case 1. A 41-year-old woman with no relevant medical history presented with a progressive generalized headache, blurred vision, disorientation, gait disturbance, and difficulty concentrating. The physical examination revealed only diminished attention and anxiety with normal language fluency. The MR imaging showed diffuse DWI hyperintensity of the entire corpus callosum with extension to the fornices with low ADC values (461$550 \mathrm{~mm}^{2} / \mathrm{s} \times 10^{-6}$ ) in the structures mentioned above (Fig $1 A$, $-B)$. The patient had been consuming a thermogenic dietary supplement for 1 month. The supplement was suspended, and the patient was administered glucocorticoid therapy. Neurologic symptoms improved 5 days later. Meanwhile, the MR imaging alterations completely resolved on a control MR imaging after 6 weeks.

Case 2. A 30-year-old woman with no relevant medical history was admitted with 2-day distal ascending paresthesia associated with a generalized headache. The physical examination findings were normal. MR imaging showed a hyperintense DWI signal from the rostrum to the splenium of the corpus callosum with bilateral involvement of the cerebral commissures and the fornix columns (Fig 1C), with decreased ADC values (351-475 $\mathrm{mm}^{2} / \mathrm{s} \times$ $\left.10^{-6}\right)$. The patient revealed a 1 -month history of thermogenic intake. The supplement was suspended, and glucocorticoid was administered. All neurologic symptoms improved 3 days later. The patient was then discharged; she missed follow-up appointments.

Case 3. A 37-year-old woman with no relevant medical history presented with 7-month distal ascending paresthesia. The physical examination did not reveal any motor involvement, only mild hyporeflexia. A lumbar puncture was performed, which revealed high protein levels $(125 \mathrm{mg} / \mathrm{dL})$ with no leukocyte count. Intravenous immunoglobulin was administered on the basis of suspected Guillain-Barré syndrome; however, the patient's symptoms persisted after 2 months of treatment. The MR imaging 


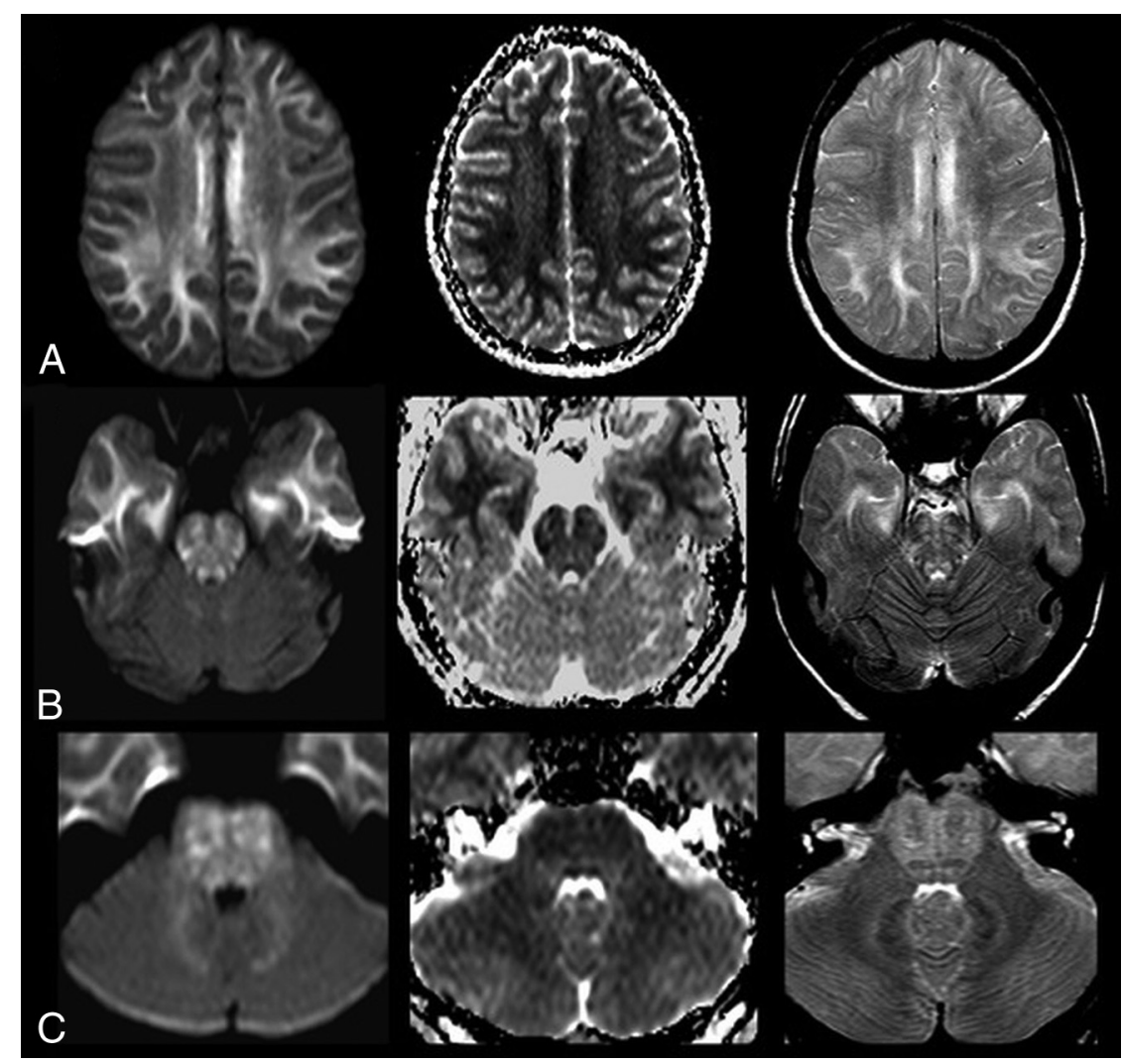

FIG 2. CLOCCs caused by thermogenic dietary supplements. Set of images from case 4 that show cytotoxic edema extending to the peripheral cerebral white matter ( $\operatorname{row} A$ ), brain stem (row $B$ ), and dentate nucleus of the cerebellum (row C). Left column, DWI trace images. Middle column, ADC map. Right column, T2WI.

Radiologic and demographic data for each patient

\begin{tabular}{lccclc}
\hline & \multicolumn{3}{c}{$\begin{array}{c}\text { Duration of } \\
\text { Thermogenic } \\
\text { Use (mo) }\end{array}$} & Affected Brain Regions & $\begin{array}{c}\text { ADC Values } \\
\text { (Range) } \\
\left(\mathbf{m m}^{2} / \mathbf{s} \times \mathbf{~ 1 0}^{-6} \text { ) }\right.\end{array}$ \\
\hline Case 1 & 41 & 20.1 & 1 & CC, FR & $461-550$ \\
Case 2 & 30 & 23.7 & 1 & CC, FR, CO & $351-475$ \\
Case 3 & 37 & 23.3 & 7 & CC, FR, CO, WM & $485-524$ \\
Case 4 & 23 & 19.2 & 3 & CC, CO, BS, DN, ON & $284-370$ \\
Case 5 & 43 & 19.5 & 18 & CC, WM, DN, ON, CH, OT & $405-440$ \\
Case 6 & 25 & 19.7 & 2 & CC, WM, BS, DN, CH & $194-275$ \\
& Mean = 33 & Mean = 21.01 & Mean $=5.3$ & & Mean $=400$ \\
\hline
\end{tabular}

Note:-BMI indicates body mass index; CC, corpus callosum; FR, fornices; CO, cerebral commissures; BS, cerebral brain stem; DN, dentate nucleus of the cerebellum; ON, optic nerves; $\mathrm{CH}$, chiasm; OT, optic tracts. commissures, peripheral cerebral white matter (Fig 2A), brain stem (Fig 2B), and dentate nucleus of the cerebellum (Fig $2 C)$, with enlargement and hyperintensity of the optic nerves on DWI, with low ADC values $\left(284-370 \mathrm{~mm}^{2} / \mathrm{s} \times 10^{-6}\right)$. Loss of gyral cerebral morphology was also present with diminished convexity of the subarachnoid space. The patient had a 3-month history of thermogenic supplement intake. Intravenous glucocorticoid was administered, and notably, the neurologic symptoms improved the day after. The patient was discharged and had no follow-up imaging.

Case 5. A 43-year-old woman with a medical history of ischemic stroke 8 years before the onset of new symptoms presented with a 4-month history of blurred vision, intermittent frontal headache, and frequent falling. The physical examination revealed gait ataxia, bilateral dysmetria, and diminished pupillary light reflex. MR imaging showed increased volume and a hyperintense DWI signal of the corpus callosum with decreased ADC values (405$\left.440 \mathrm{~mm}^{2} / \mathrm{s} \times 10^{-6}\right)$. Additionally, the hemispheric white matter showed increased signal in T2 and FLAIR sequences, with extension to the brain stem and dentate nucleus and compromised optic nerves, chiasm, and optic tracts. The patient received glucocorticoid therapy and then revealed continuous thermogenic supplement intake for 18 months. The neurologic symptoms improved 5 days after supplement discontinuation. Despite that, the MR imaging alterations were only partially resolved up to 10 months later in serial control studies (Fig $3 A,-B$ ). showed a hyperintense DWI signal of the corpus callosum, cerebral commissures, and fornices, with extension to the periventricular cerebral white matter, associated with discrete, low ADC values $\left(485-524 \mathrm{~mm}^{2} / \mathrm{s} \times 10^{-6}\right)$. The patient then revealed a 7-month history of thermogenic supplement intake. Neurologic symptoms improved 3 days after supplement discontinuation. A control MR imaging performed after 2 months showed significant reduction of the DWI brain abnormalities, with only a mild bilateral symmetric edema on the hippocampi.

Case 4. A 23-year-old woman with no relevant medical history presented with a 7-day progressive generalized headache associated with blurred vision and left-arm weakness. The physical examination revealed left-arm paresis only. MR imaging showed extensive cytotoxic edema of the corpus callosum and cerebral
Case 6. A 25-year-old woman with no relevant medical history presented with a 2-month progressive, generalized, oppressive headache associated with photophobia and phonophobia that did not improve with oral painkillers. The physical examination revealed papilledema and gait disturbances. The MR imaging showed a DWI hyperintense signal with decreased ADC values $\left(194-275 \mathrm{~mm}^{2} / \mathrm{s} \times 10^{-6}\right)$ of the callosal commissure, brain stem, dentate nucleus of the cerebellum, optic chiasm, and bilateral frontal, parietal, and occipital white matter. The patient revealed a 2-month history of thermogenic supplement intake. She received supportive treatment with osmotic therapy, and neurologic symptoms improved 2 days after supplement discontinuation, whereas the MR imaging findings completely resolved after 22 months (Fig 3C, $-D$ ). 


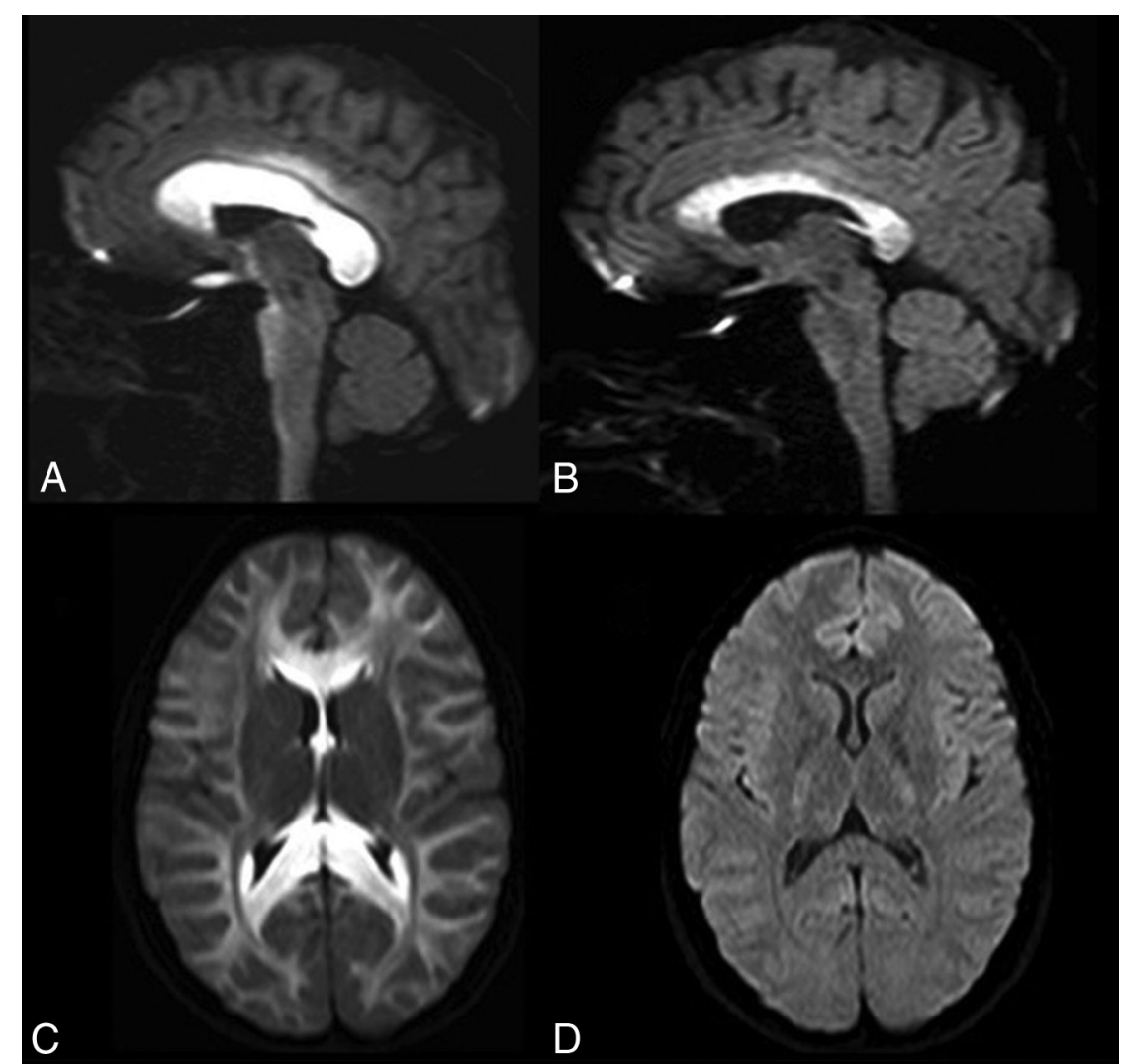

FIG 3. CLOCCs caused by thermogenic dietary supplements. A, Sagittal DWI shows an enlarged edematous corpus callosum with involvement of the optic chiasm in case 5. B, Sagittal DWI from an MR imaging control study of the same patient obtained 8 weeks after the discontinuation of the thermogenic dietary supplement shows a decrease in volume and signal hyperintensity of the corpus callosum and visual pathway, representing partial recovery. $C$, The initial MR imaging shows extensive cytotoxic edema of the corpus callosum and cerebral white matter in case 6.D, The MR imaging of the same patient obtained 22 months later shows complete recovery with normal brain findings on DWI.

\section{DISCUSSION}

Toxic leukoencephalopathy can be caused by different exogenous agents, and although the clinical and imaging presentation may be severe, it can be reversible. Some of these substances have a predilection for the corpus callosum. ${ }^{5}$ Thermogenics are readily available on the World Wide Web as overthe-counter dietary supplements. Herbal preparations consumed by this series of patients shared a combination of botanical and biochemical ingredients like bitter melon ( $\mathrm{Mo}$ mordica charantia), green tea, horsetail herb (Equisetum), bearberry (Arctostaphylos uva-ursi), olive, juniper, ginger, yerba mate, grape, cempasuchil flower (Tagetes erecta) pigment, L-carnitine tartrate, and microcrystalline cellulose among unspecified others. Some substances found in these compounds, especially the combination of high doses of caffeine and ephedrine analogs, have an action mechanism that increases the rate of fat oxidation and lipolysis with reduced glycogen metabolism. ${ }^{6,7}$ Some of these plant molecules have other in vitro metabolic activities. For example, bitter melon is reported to be a bioactive insulin-like structure, which has exhibited a moderate effect on the reduction of blood glucose levels in clinical studies. Most interesting, CLOCCs in adult patients with acute hypoglycemia have a common predilection for the posterior corpus callosum and tracts of deep white matter. ${ }^{8,9}$ Thus, in this series, the corpus callosum was more extensively affected in all patients. Bitter melon has also been associated with significant adverse effects, which include anxiety, insomnia, and cardiovascular disorders, caused by stimulation of the central nervous system. $^{9}$

The term "leukoencephalopathy" encompasses a spectrum of diseases that predominantly involve the white matter structure as a result of exposure to a variety of exogenous agents; known causes are chemo- and immunotherapy, antibiotics, and environmental and infectious agents. ${ }^{10}$ In some cases, the cause is unknown. ${ }^{11,12}$ Although the central nervous system is naturally protected by the blood-brain barrier, some of these agents are known to interfere with or damage this barrier, allowing access of potentially threatening substances to neural tissue. Other agents are fat-soluble; this feature facilitates their distribution into the central nervous system. ${ }^{13}$ Acute toxic leukoencephalopathy may have a severe extension and may be reversible both clinically and radiologically. ${ }^{14}$ Clinical presentation is nonspecific, depending on the distribution of edema, often including neurologic decline, distal paresthesia, personality changes, memory alterations, headache, and other focal neurologic signs. ${ }^{15}$

Damage usually involves white matter tracts from the periventricular-to-subcortical areas, though other metabolically active areas may be susceptible to neurotoxicity, including the deep gray matter and the brain stem. Therefore, these structures may show changes in acute toxic leukoencephalopathy imaging. Regarding its pathophysiology, theories posit that cytotoxic edema is caused by the increased water molecule influx in neurons and astrocytes. The splenium is the most affected area of the corpus callosum because of its high density of glutamate, and cytokine and drug receptors. ${ }^{4,5,14,16,17}$

Drug agencies around the world have issued health warnings against some of the thermogenic dietary supplements consumed by our series of patients, including the Comisión Federal para la Protección contra Riesgos Sanitarios (COFEPRIS) in Mexico and Agencia Española de Medicamentos y Productos Sanitarios (AEMPS) in Spain and this motivated a recall by the FDA in the United States. ${ }^{18,19}$ Nonetheless, these products can still be easily purchased on-line without a prescription under various brand names. $3,18,19$

All the patients in our series had normal body weight with normal body mass index values at the time of examination. They did not indicate that previous obesity or being significantly overweight had motivated them to consume the dietary supplements, 
which could be a risk factor for developing CLOCCs. However, due to the limited number of cases, we cannot make assumptions on this issue.

Two of our patients were on antiepileptic or antidepressant medication, both of which have been reported to cause drug-related CLOCCs. ${ }^{4}$ The fact that the other 4 patients lack this risk factor makes this association of a comorbid condition less plausible. Even so, there is a need for further analysis on a larger number of patients to determine whether there is any relationship. One patient had a history of social alcohol consumption but no chronic alcoholism that would raise the suspicion of Marchiafava-Bignami disease as an additional metabolic cause for developing CLOCCs. ${ }^{4,5,20}$

As observed in our series, the clinical symptoms of CLOCCs caused by thermogenic dietary supplements are frequently subtle and may not always prompt subjects to seek medical attention, making it possible that a significant number of cases occur undetected.

Although the clinical neurologic signs and symptoms improved rapidly after the withdrawal of the supplement, the white matter lesions on MR imaging reduced gradually and progressively resolved in a highly variable fashion ( 6 weeks to 22 months), seemingly dependent on the extension of leukoencephalopathy. There may be signs of peripheral nerve involvement as in case 3, but there is not enough evidence to confirm this possibility. Additionally, we do not know if there is any potential long-term neurologic damage resulting from thermogenic consumption as in other toxic leukoencephalopathies. ${ }^{4}$

Given the lack of regulation in the manufacturing process of these herbal dietary supplements, it is difficult to know if the brain toxicity was caused by a single defective batch, though its widespread presentation for a long period suggests otherwise. Also, the information provided on the label of these products is often incomplete. For example, the labels do not state the specific parts of the plants used. Because the concentration of each active compound could be highly variable, this feature can result in possible toxic combinations not yet identified. ${ }^{20}$ It is conceivable that incorrect handling of these ingredients or contamination due to inadequate supervision during fabrication could lead to potentially harmful products.

\section{CONCLUSIONS}

Thermogenics are widely available but potentially harmful dietary supplements that have prompted warnings and recalls by international drug regulation agencies; nonetheless, they can still be easily purchased on-line. These herbal preparations being labeled as natural does not mean that they are entirely safe. When used without medical supervision, they can produce toxic neurologic side effects such as CLOCCs. It is not known whether a normal body mass index before consumption could play a role in developing toxicity or if there could be any interactions between the dietary supplements and other substances, such as prescription drugs or over-the-counter medications. With this publication, we add 6 new cases to the 2 previously reported cases of toxic leukoencephalopathy caused by thermogenic dietary supplements and to the toxic etiologies of CLOCCs, making the medical community aware of the clinical and radiologic characteristics of this potential complication.

\section{REFERENCES}

1. Blanck HM, Serdula MK, Gillespie C, et al. Use of nonprescription dietary supplements for weight loss is common among Americans. J Am Diet Assoc 2007;107:441-47 CrossRef Medline

2. Hoffman JR, Kang J, Ratamess NA, et al. Thermogenic effect of an acute ingestion of a weight loss supplement. J Int Soc Sports Nutr 2009;6:1 CrossRef Medline

3. Olivas-Chacon C, Treviño-García M, Chua-Tuan J, et al. Leukoencephalopathic changes on magnetic resonance imaging associated with a thermogenic dietary supplement (Thermatrim). Proc (Bayl Univ Med Cent) 2015;28:389-91 CrossRef Medline

4. Starkey J, Kobayashi N, Numaguchi Y, et al. Cytotoxic lesions of the corpus callosum that show restricted diffusion: mechanisms, causes, and manifestations. Radiographics 2017;37:562-76 CrossRef Medline

5. Rimkus CM, Andrade CS, Leite C, et al. Toxic leukoencephalopathies, including drug, medication, environmental, and radiationinduced encephalopathic syndromes. Semin Ultrasound CT MR 2014;35:97-117 CrossRef Medline

6. Diepvens K, Westerterp KR, Westerterp-Plantenga MS. Obesity and thermogenesis related to the consumption of caffeine, ephedrine, capsaicin, and green tea. Am J Physiol Regul Integr Comp Physiol 2007;292:R77-85 CrossRef Medline

7. Liu AG, Smith SR, Fujioka K, et al. The effect of leptin, caffeine/ephedrine, and their combination upon visceral fat mass and weight loss. Obesity (Silver Spring) 2013;21:1991-96 CrossRef Medline

8. Evans JL. Diet, botanical and nutritional treatments for type 2 diabetes. 2003. https://www.endotext.org/. Accessed June 13, 2019

9. da Silva WV, de Andrade Gomes Silva MI, Tavares Toscano L, et al. Supplementation prevalence and adverse effects in physical exercise practitioners. Nutr Hosp 2014;29:158 - 65 CrossRef Medline

10. Sathananthasarma $P$, Weeratunga $P N$, Chang T. Reversible splenial lesion syndrome associated with dengue fever: a case report. $B M C$ Res Notes 2018;11:9-12 CrossRef Medline

11. Singh P, Gogoi D, Vyas S, et al. Transient splenial lesion: further experience with two cases. Indian J Radiol Imaging 2010;20:254-57 CrossRef Medline

12. Cho JS, Ha SW, Han YS, et al. Mild encephalopathy with reversible lesion in the splenium of the corpus callosum and bilateral frontal white matter. J Clin Neurol 2007;3:53-56 CrossRef Medline

13. Zheng W. Neurotoxicology of the brain barrier system: new implications. J Toxicol Clin Toxicol 2001;39:711-19 CrossRef Medline

14. McKinney AM, Kieffer SA, Paylor RT, et al. Acute toxic leukoencephalopathy: potential for reversibility clinically and on MRI with diffusion weighted and FLAIR imaging. AJR Am J Roentgenol 2009;193:192-206 CrossRef Medline

15. Hagan IG, Burney K. Radiology of recreational drug abuse. Radiographics 2007;27:919-40 CrossRef Medline

16. Hassel B, Boldingh KA, Narvesen C, et al. Glutamate transport, glutamine synthetase and phosphate-activated glutaminase in rat CNS white matter: a quantitative study. J Neurochem 2003;87:230-37 CrossRef Medline

17. Domercq M, Matute C. Expression of glutamate transporters in the adult bovine corpus callosum. Brain Res Mol Brain Res 1999;67:296302 CrossRef Medline

18. Cofepris. LA COFEPRIS ALERTA SOBRE EL PRODUCTO THERMATRIM. Secretaría de Salud: Comunicado de prensa 2014. https://www.gob.mx/salud/prensa/la-cofepris-alerta-sobre-elproducto-thermatrim. Accessed June 13, 2019

19. Food Alert Network. Efectos adversos relacionados con el consumo del complemento alimenticio (Pura Alegría) procedente de México 2015. http://www.aecosan.msssi.gob.es/AECOSAN/web/seguridad_ alimentaria/ampliacion/complemento_alimenticio_PURA_ ALEGRIA.htm. Accessed June 13, 2019

20. Kakkar C, Prakashini K, Polnaya A. Acute Marchiafava Bignami disease: clinical and serial MRI correlation. BMJ Case Rep 2014;2014 CrossRef Medline 